PROCEEDINGS OF THE

AMERICAN MATHEMATICAL SOCIETY

Volume 134, Number 4, Pages 1143-1151

S 0002-9939(05)08203-1

Article electronically published on September 28, 2005

\title{
ON DIFFERENTIABILITY PROPERTIES OF TYPICAL CONTINUOUS FUNCTIONS AND HAAR NULL SETS
}

\author{
L. ZAJÍČEK
}

(Communicated by David Preiss)

\begin{abstract}
Let $D\left(D^{*}\right)$ be the set of all continuous functions $f$ on $[0,1]$ which have a derivative $f^{\prime}(x) \in \mathbf{R}\left(f^{\prime}(x) \in \mathbf{R}^{*}\right.$, respectively) at least at one point $x \in(0,1)$. B. R. Hunt (1994) proved that $D$ is Haar null (in Christensen's sense) in $C[0,1]$.

In the present article it is proved that neither $D^{*}$ nor its complement is Haar null in $C[0,1]$. Moreover, the same assertion holds if we consider the approximate derivative (or the "strong" preponderant derivative) instead of the ordinary derivative; these results are proved using a new result on typical (in the sense of category) continuous functions, which is of interest in its own right.
\end{abstract}

\section{INTRODUCTION}

In what follows we denote by $C[0,1]$ the space of all continuous real functions on $[0,1]$ equipped with the supremum norm $\|$.$\| . We put \mathbf{R}^{*}=\mathbf{R} \cup\{-\infty, \infty\}$.

Let $D\left(D^{*}\right)$ be the set of all $f \in C[0,1]$ which has a derivative $f^{\prime}(x) \in \mathbf{R}$ $\left(f^{\prime}(x) \in \mathbf{R}^{*}\right.$, respectively) at least at one point $x \in(0,1)$. It was shown by Banach, Mazurkiewicz and Saks (see e.g. [J1]) that both $D$ and $D^{*}$ are negligible in the topological sense - they are of the first category in $C[0,1]$. B. R. Hunt [H] (using another terminology) proved that $D$ is negligible also in a measure sense - it is Haar null (in Christensen's sense) in $C[0,1]$. (In the terminology of $[\underline{\mathrm{H}}], D$ is a shy set and its complement is a prevalent set in $C[0,1]$.)

Recall (see e.g. $[\mathrm{BL})$ ) that a Borel subset $A$ of $C[0,1]$ is said to be Haar null if there exists a Radon probability measure $\mu$ on $C[0,1]$ such that $\mu(A+f)=0$ for each $f \in C[0,1]$. (If $A$ is not Borel, we will say that $A$ is Haar null, if $A$ is contained in a Borel Haar null set.)

We note that there is no non-zero $\sigma$-finite translation invariant measure on a separable infinite-dimensional Banach space $X$ (see [BL pp. 130, 143]) and that the notion of Haar null sets on $X$ is a natural (translation invariant) generalization of the notion of Lebesgue null sets which has a number of interesting applications. For other interesting null sets see [BL] (Gaussian null sets) and [LP] ( $\Gamma$-null sets).

Received by the editors March 5, 2004 and, in revised form, November 9, 2004

2000 Mathematics Subject Classification. Primary 26A27; Secondary 28C20.

Key words and phrases. Typical continuous function, Haar null set, nowhere differentiable function, approximative derivative, preponderant derivative.

This research was supported by MSM 113200007, GAČR 201/00/0767 and GAČR 201/03/0931

(C)2005 American Mathematical Society Reverts to public domain 28 years from publication 
Notice that Hunt's result can be obtained using a very slight modification of the well-known argument showing that almost all trajectories of one-dimensional Brownian motion are nowhere differentiable (see [HZ]).

Further, note that Hunt's result was recently improved by J. Kolár [K1] in several directions. In particular, Kolár proved that the set of all $f \in C[0,1]$ which have a finite one-sided approximate derivative at least at one point $x \in(0,1)$ is Haar null.

In the proof of Theorem 6 we will see that the Arzelà-Ascoli theorem easily implies that $D^{*}$ is not Haar null. Using also one of Jarník's results from [J1, it is easy to prove (see Remark 2 (ii)) that $C[0,1] \backslash D^{*}$ is not Haar null as well. Thus, if we allow infinite derivatives, the "Haar null case" differs from the "category case": neither nowhere differentiable nor somewhere differentiable functions are negligible in Christensen's sense. This result was stated in [Z2, Proposition 3.2] without a proof. (In the case of infinite one-sided derivatives, the category and measure cases are similar; see Remark 3 (i).)

Moreover, we prove that the same result holds if we consider the approximate derivative (or the "strong" preponderant derivative) instead of the ordinary derivative. For the proof we need the main result of the second section (Theorem 11) on typical (in the sense of category) continuous functions which improves Jarník's above-mentioned result from [J1] and is of interest in its own right.

We use the following terminology.

The symbol $\lambda$ denotes Lebesgue measure on $\mathbf{R}$. The open ball with center $x \in C[0,1]$ and radius $r>0$ will be denoted by $B(x, r)$.

For a Lebesgue measurable set $E \subset \mathbf{R}$ and $x \in \mathbf{R}$ we define the upper right, upper left and upper symmetric densities $d^{+}(E, x), d^{-}(E, x), d^{s}(E, x)$ of $E$ at $x$ by

$$
\begin{gathered}
d^{+}(E, x):=\varlimsup_{h \rightarrow 0+} \frac{\lambda(E \cap(x, x+h))}{h}, d^{-}(E, x):=\varlimsup_{h \rightarrow 0+} \frac{\lambda(E \cap(x-h, x))}{h}, \\
d^{s}(E, x):=\varlimsup_{h \rightarrow 0+} \frac{\lambda(E \cap(x-h, x+h))}{2 h} .
\end{gathered}
$$

Using the lower limit, the lower unilateral densities $d_{+}(E, x), d_{-}(E, x)$ are defined.

The symbols ap- $\lim _{x \rightarrow a} f(x)$ and $f_{\text {ap }}^{\prime}$ denote the approximate limit and the approximate derivative, respectively.

We say that a function $f$ has at a point $a \in \mathbf{R}$ the preponderant derivative $f_{\mathrm{pr}}^{\prime}(a)=A \in \mathbf{R}^{*}$ if there exists a Lebesgue measurable set $E \subset \mathbf{R}$ such that $d_{+}(E, x)>1 / 2, d_{-}(E, x)>1 / 2$ and $\lim _{x \rightarrow a, x \in E}(f(x)-f(a)) /(x-a)=A$.

\section{A NEW RESUlt ON TYPiCAL CONTINUOUS FUnCtions}

Results of V. Jarník [J1] imply that, for a typical $f \in C[0,1]$, at each point $x \in(0,1)$,

$$
\bar{D}^{+} f(x)=\infty \text { or } \bar{D}^{-} f(x)=\infty,
$$

where $\bar{D}^{+} f(x), \bar{D}^{-} f(x)$ are upper one-sided Dini derivatives.

In fact, Jarník in J1] proved a stronger general result. He considered "generalized Dini derivatives" with respect to a function $\varphi \in \Phi$, where $\Phi$ is the system of all odd functions $\varphi: \mathbf{R} \rightarrow \mathbf{R}$ such that $\varphi(h)>0$ for $h>0, \varphi(0)=0$ and $\varphi$ is continuous at 0 . Given $\varphi \in \Phi$, we define

$$
\bar{D}_{\varphi}^{+} f(x)=\limsup _{h \rightarrow 0+} \frac{f(x+h)-f(x)}{\varphi(h)} .
$$


The other derivatives $\underline{D}_{\varphi}^{+} f(x), \bar{D}_{\varphi}^{-} f(x), \underline{D}_{\varphi}^{-} f(x)$ are defined in the obvious way; they clearly coincide with the ordinary Dini derivatives in the case $\varphi(h)=h$.

By [J1, for an arbitrary fixed $\varphi \in \Phi$, we can replace (10) by

$$
\bar{D}_{\varphi}^{+} f(x)=\infty \text { or } \bar{D}_{\varphi}^{-} f(x)=\infty .
$$

In [J2 Jarník considered derived numbers of $f$ at $x$ with (right, left, symmetric) density $\alpha$. (We use here the terminology which was introduced by Jarník in [J3].) Now we will consider the natural joint generalization of Jarník's above-mentioned concepts.

Definition 1. Let $\varphi \in \Phi, \alpha \geq 0$ and $d \in \mathbf{R}^{*}$ be given. We say that $d$ is a derived number of (a real function of a real variable) $f$ at $x \in \mathbf{R}$ with respect to $\varphi$ with upper right (upper left; upper symmetric; upper one-sided) density $\alpha$ if there exists a measurable set $E \subset \mathbf{R}$ such that $d^{+}(E, x) \geq \alpha\left(d^{-}(E, x) \geq \alpha ; d^{s}(E, x) \geq \alpha\right.$; $d^{-}(E, x) \geq \alpha$ or $\left.d^{+}(E, x) \geq \alpha\right)$ and $\lim _{y \rightarrow x, y \in E} \frac{f(y)-f(x)}{\varphi(y-x)}=d$. If $\varphi(t)=t$, "with respect to $\varphi^{\prime \prime}$ is omitted.

Jarník in J2 proved that (1) can be replaced by the stronger statement

$\infty$ is a derived number of $f$ at $x$ with upper symmetric density $1 / 2$.

I do not know whether the joint natural generalization of all Jarník's abovementioned results holds (see Remark 1 (iii)). So we will prove only the following weaker version, which is sufficient for the applications in the following section.

Theorem 1. Let $\varphi \in \Phi$ be given. Then, for a typical $f \in C[0,1]$, both $\infty$ and $-\infty$ are derived numbers of $f$ at each $x \in[0,1]$ with respect to $\varphi$ with upper one-sided density $1 / 2$.

Remark 1. (i) Theorem 1 clearly improves Jarník's result about (2).

(ii) For $\varphi(t)=t$, Jarník's result about (3) easily implies Theorem 1 but not vice versa.

(iii) It is not clear whether Theorem 1 remains true if we replace "upper one-sided density $1 / 2$ " by "upper symmetric density $1 / 2$ ".

Before proving Theorem 1 we formulate two lemmas, omitting their easy and straightforward proofs.

Lemma 2. Let $f$ be a function defined on $[0,1], \varphi \in \Phi, x \in[0,1]$ and suppose the following condition holds:

(C) For each $q>0$ there exists a closed interval $J \subset[0,1] \backslash\{x\}$ such that $|J|<1 / q, \frac{|J|}{\operatorname{dist}(x, J)}>1-1 / q$ and $\frac{f(y)-f(x)}{\varphi(y-x)}>q$ for each $y \in J$.

Then $\infty$ is a derived number of $f$ at $x$ with respect to $\varphi$ with upper one-sided density $1 / 2$.

Lemma 3. Let $K_{n}>0, n=1,2, \ldots$, and $\lim _{n \rightarrow \infty} K_{n}=\infty$. Then there exists a sequence $\left(f_{n}\right)_{n=1}^{\infty}$ which is dense in $C[0,1]$ where $f_{n}$ is Lipschitz with the constant $K_{n}$ for each $n$.

Proof of Theorem 1. Put $s_{n}:=\sup _{t \in(0,1 / n]} \varphi(t)$. Since $s_{n} \rightarrow 0$, we can choose a sequence $v_{n}>0, n=1,2, \ldots$, such that $v_{n} \rightarrow 0$ and

$$
\max \left(s_{n}, 1 / n\right)=o\left(v_{n}\right), n \rightarrow \infty .
$$


Since $1 / n=o\left(v_{n}\right), n \rightarrow \infty$, we can choose a sequence $K_{n}>0, n \in \mathbf{N}$, such that

$$
K_{n} \rightarrow \infty \text { and } \frac{K_{n}}{n}=o\left(v_{n}\right), n \rightarrow \infty
$$

Further, put

$$
\varepsilon_{n}=\frac{v_{n}}{n} \quad \text { and } \quad A_{n}=\frac{1}{s_{n}}\left(\frac{v_{n}}{2}-\frac{K_{n}}{n}-2 \varepsilon_{n}\right) .
$$

Since $s_{n}>0$, by (44) and (5) we have

$$
A_{n} \rightarrow \infty \text {. }
$$

By Lemma 3 we can choose a sequence $\left(f_{n}\right)_{1}^{\infty}$ which is dense in $C[0,1]$ and such that each $f_{n}$ is Lipschitz with the constant $K_{n}$.

Further consider, for each positive integer $n \geq 3$, the function $g_{n} \in C[0,1]$ with the following properties:

- If $x \in\left[\frac{k}{2 n}, \frac{k+1}{2 n}-\frac{1}{n^{2}}\right], k=0, \ldots, 2 n-1$, then $g_{n}(x)=0$ if $k$ is even and $g_{n}(x)=v_{n}$ if $k$ is odd.

- $g_{n}(1)=0$.

- $g_{n}$ is linear on each interval $\left[\frac{k+1}{2 n}-\frac{1}{n^{2}}, \frac{k+1}{2 n}\right], k=0, \ldots, 2 n-1$.

Now put $B_{n}:=B\left(f_{n}+g_{n}, \varepsilon_{n}\right)$ and

$$
P:=\limsup _{n \rightarrow \infty} B_{n}=\bigcap_{m=3}^{\infty} \bigcup_{n=m}^{\infty} B_{n} .
$$

Since $\left\|g_{n}\right\| \leq v_{n}$ and $v_{n} \rightarrow 0$, it is easy to see that the sequence $\left(f_{n}+g_{n}\right)_{n=1}^{\infty}$ is dense in $C[0,1]$ and consequently $\bigcup_{n=p}^{\infty} B_{n}$ is a dense open set for each $p \geq 3$. Therefore $P$ is a residual set in $C[0,1]$.

Now consider arbitrary $f \in P, x \in[0,1)$ and $q>0$. By (6) we can choose $n \geq 3$ such that $f \in B_{n}$ and

$$
n>4 q, A_{n}>q, 1-x>\frac{1}{n} .
$$

Since $1-x>1 / n$, there exists $p \in\{0, \ldots, n-2\}$ such that $x \in[p / n,(p+1) / n)$. We will distinguish three possible cases.

(a) $x \in\left[\frac{p}{n}, \frac{2 p+1}{2 n}-\frac{1}{2 n^{2}}\right)$.

Then clearly $g_{n}(x) \leq \frac{v_{n}}{2}$ and we put $J:=\left[\frac{2 p+1}{2 n}, \frac{p+1}{n}-\frac{1}{n^{2}}\right]$. Obviously $|J|<$ $\frac{1}{2 n}<\frac{1}{q}$ and

$$
\frac{|J|}{\operatorname{dist}(x, J)} \geq\left(\frac{1}{2 n}-\frac{1}{n^{2}}\right) /\left(\frac{1}{2 n}\right)=1-\frac{2}{n}>1-\frac{1}{q} .
$$

Further, for each $y \in J$ we have $g_{n}(y)=v_{n}, 0<y-x \leq \frac{1}{n}$. Therefore, using also $f \in B_{n}$, the definitions of $A_{n}, s_{n}$ and (7), we obtain

$$
\begin{aligned}
\frac{f(y)-f(x)}{\varphi(y-x)} & \geq \frac{g_{n}(y)-g_{n}(x)}{\varphi(y-x)}-\frac{\left|f_{n}(y)-f_{n}(x)\right|}{\varphi(y-x)}-\frac{2 \varepsilon_{n}}{\varphi(y-x)} \\
& \geq \frac{1}{\varphi(y-x)}\left(\frac{v_{n}}{2}-\frac{K_{n}}{n}-2 \varepsilon_{n}\right)=\frac{A_{n} s_{n}}{\varphi(y-x)} \geq A_{n}>q .
\end{aligned}
$$

(b) $x \in\left[\frac{2 p+1}{2 n}-\frac{1}{2 n^{2}}, \frac{2 p+2}{2 n}-\frac{1}{2 n^{2}}\right]$.

Then $g_{n}(x) \geq v_{n} / 2$ and we put $J:=\left[\frac{p}{n}, \frac{2 p+1}{2 n}-\frac{1}{n^{2}}\right]$. We have $|J|<\frac{1}{2 n}<\frac{1}{q}$ and

$$
\frac{|J|}{\operatorname{dist}(x, J)} \geq\left(\frac{1}{2 n}-\frac{1}{n^{2}}\right) /\left(\frac{1}{2 n}+\frac{1}{n^{2}}\right)=\frac{n-2}{n+2}=1-\frac{4}{n+2}>1-\frac{1}{q} \text {. }
$$


For each $y \in J$ we have $g_{n}(y)=0,0<x-y \leq \frac{1}{n}$. Thus, as in case (a), we similarly obtain

$$
\frac{f(y)-f(x)}{\varphi(y-x)}=\frac{f(x)-f(y)}{\varphi(x-y)} \geq A_{n}>q .
$$

(c) $x \in\left[\frac{p+1}{n}-\frac{1}{2 n^{2}}, \frac{p+1}{n}\right)$.

Then $g_{n}(x) \leq v_{n} / 2$ and we put $J:=\left[\frac{2 p+3}{2 n}, \frac{p+2}{n}-\frac{1}{n^{2}}\right] \subset[0,1]$. We have $|J|<$ $\frac{1}{2 n}<\frac{1}{q}$ and

$$
\frac{|J|}{\operatorname{dist}(x, J)} \geq\left(\frac{1}{2 n}-\frac{1}{n^{2}}\right) /\left(\frac{1}{2 n}+\frac{1}{n^{2}}\right)>1-\frac{1}{q} .
$$

For each $y \in J$ we have $g_{n}(y)=v_{n}, 0<y-x \leq 1 / n$. So, as in case (a), $\frac{f(y)-f(x)}{\varphi(y-x)}>q$. Thus we have verified condition (C) of Lemma 2] consequently $\infty$ is a derived number of $f$ at $x$ with respect to $\varphi$ with upper one-sided density $1 / 2$.

Since the mapping $f \rightarrow-f$ is a homeomorphism $C[0,1]$ onto $C[0,1]$, it follows that, for a typical $f \in C[0,1],-\infty$ is a derived number of $f$ at $x$ with respect to $\varphi$ with upper one-sided density $1 / 2$ at each $x \in[0,1)$. Using also the homeomorphism $C[0,1]$ onto $C[0,1]$, which assigns to a function $f(x)$ the function $f(1-x)$, we obtain the assertion of the theorem.

\section{Sets of Functions Which have SOMEWhere AN INFINITE DERIVATIVE AND HAAR NULL SETS}

To prove that a set $S \subset C[0,1]$ is not Haar null, we will use the following almost obvious well-known lemma.

Lemma 4. Let $S \subset C[0,1]$ have the following property:

(T) For each compact set $K \subset C[0,1]$ there exists $g \in C[0,1]$ such that $g+K \subset S$.

Then $S$ is not Haar null.

Proof. Suppose on the contrary that $S$ is Haar null. Then there exists a Radon probability measure $\mu$ on $C[0,1]$ such that $\mu(S+f)=0$ for each $f \in C[0,1]$. Choose a compact set $K \subset C[0,1]$ with $\mu(K)>0$ and $g \in C[0,1]$ such that $g+K \subset S$. Then for $f:=-g$ we have $K \subset S+f$ and therefore $\mu(K)=0$; a contradiction.

We will also need the following lemma. Its easy standard proof is omitted.

Lemma 5. Let $\eta$ be a non-negative and non-decreasing function on $(0, \infty)$ such that $\lim _{t \rightarrow 0+} \eta(t)=0$. Then there exists a continuous increasing function $\varphi \in \Phi$ such that $\lim _{t \rightarrow 0+} \eta(t) / \varphi(t)=0$.

There are many natural properties $P$ of functions from $C[0,1]$ such that neither $\{f \in C[0,1]: f$ has $P\}$ nor $\{f \in C[0,1]: f$ has not $P\}$ is Haar null. Such properties will be called $H$-ambivalent properties. Notice that interesting examples of $H$-ambivalent properties (in spaces different from $C[0,1]$ ) are given in $\mathrm{D}$.

Using this terminology, we can formulate our main result in the following form.

Theorem 6. Let $a \in(0,1)$ be given. Then the following properties of $f \in C[0,1]$ are $H$-ambivalent:

$\left(P_{1}\right) \quad f^{\prime}(x)=\infty$ at some $x \in(0,1)$.

$\left(P_{2}\right) \quad f^{\prime}(x) \in \mathbf{R}^{*}$ exists at some $x \in(0,1)$.

$\left(P_{3}\right) \quad f^{\prime}(a)=\infty$. 
$\left(P_{4}\right) \quad f^{\prime}(a) \in \mathbf{R}^{*}$ exists.

Moreover, denote by $\left(P_{i}^{\mathrm{ap}}\right)$ and $\left(P_{i}^{\mathrm{pr}}\right), 1 \leq i \leq 4$, the properties obtained from the properties $\left(P_{i}\right)$ by replacing the ordinary derivative $f^{\prime}$ by the approximate derivative $f_{\mathrm{ap}}^{\prime}$ and by the preponderant derivative $f_{\mathrm{pr}}^{\prime}$, respectively. Then all these properties are also $H$-ambivalent.

Proof. Since the strongest of the considered twelve properties is $\left(P_{3}\right)$ and the weakest is $\left(P_{2}^{\mathrm{pr}}\right)$, it is sufficient to prove that neither the set $A:=\left\{f: f^{\prime}(a)=\infty\right\}$ nor the set $B:=\left\{f: f_{\mathrm{pr}}^{\prime}(x) \in \mathbf{R}^{*}\right.$ exists at no point $\left.x \in(0,1)\right\}$ is Haar null.

Let an arbitrary compact set $K \subset C[0,1]$ be given. By the Arzelà-Ascoli theorem, $K$ is uniformly equicontinuous. Consequently, the function

$$
\omega(t):=\sup \{|h(x)-h(y)|: x, y \in[0,1],|x-y| \leq t, h \in K\}, \quad t \in(0, \infty),
$$

is finite, non-negative and non-decreasing; also, $\lim _{t \rightarrow 0+} \omega(t)=0$.

Using Lemma 5 for $\eta(t):=\max (\omega(t), t)$, we obtain a continuous increasing function $\varphi \in \Phi$ such that

$$
\lim _{t \rightarrow 0+} \omega(t) / \varphi(t)=0 \quad \text { and } \quad \lim _{t \rightarrow 0} \varphi(t) / t=\infty .
$$

(a) To prove that $A$ is not Haar null, it is, by Lemma 4, sufficient to find a function $g \in C[0,1]$ such that $g+K \subset A$. We can put $g(z):=\varphi(z-a), z \in[0,1]$. Indeed, if $h \in K$ and $a+t \in(0,1)$, then

$$
\begin{aligned}
\frac{(g+h)(a+t)-(g+h)(a)}{t} & =\frac{\varphi(t)+h(a+t)-h(a)}{t} \\
& =\frac{\varphi(t)}{t}\left(1+\frac{h(a+t)-h(a)}{\varphi(t)}\right) .
\end{aligned}
$$

Therefore (9) implies $(g+h)^{\prime}(a)=\infty$.

(b) To prove that $B$ is not Haar null, choose, by Theorem 1, a function $g$ such that both $\infty$ and $-\infty$ are derived numbers of $g$ at each $x \in[0,1]$ with respect to $\varphi$ with upper one-sided density $1 / 2$. By Lemma 4, it is sufficient to prove $g+K \subset B$. Suppose on the contrary that there exist $h \in K$ and $x \in(0,1)$ such that $(g+h)_{\mathrm{pr}}^{\prime}(x)=d \in \mathbf{R}^{*}$ exists. Suppose also that $d<\infty$, the case $d>-\infty$ being quite analogous. Further, without any loss of generality, suppose that $\infty$ is a derived number of $g$ at $x$ with respect to $\varphi$ with upper right density $1 / 2$.

Let $P \subset \mathbf{R}$ be a Lebesgue measurable set such that $d_{+}(P, x)>1 / 2$ and

$$
\lim _{y \rightarrow x, y \in P} \frac{(g+h)(y)-(g+h)(x)}{y-x}=d .
$$

Let $M \subset \mathbf{R}$ be a Lebesgue measurable set such that $d^{+}(M, x) \geq 1 / 2$ and $\lim _{y \rightarrow x, y \in M} \frac{g(y)-g(x)}{\varphi(y-x)}=\infty$. Since

$$
\frac{(g+h)(y)-(g+h)(x)}{y-x}=\left(\frac{g(y)-g(x)}{\varphi(y-x)}+\frac{h(y)-h(x)}{\varphi(y-x)}\right) \frac{\varphi(y-x)}{y-x},
$$

(9) implies

$$
\lim _{y \rightarrow x, y \in M} \frac{(g+h)(y)-(g+h)(x)}{y-x}=\infty .
$$


The inequalities $d^{+}(M, x) \geq 1 / 2$ and $d_{+}(P, x)>1 / 2$ clearly imply that $x$ is a right accumulation point of $M \cap P$. Therefore (11) contradicts (10).

Remark 2. (i) The proof clearly shows that Theorem 6 remains true if we write in its formulation $a \in[0,1]$ and $x \in[0,1]$ instead of $a \in(0,1)$ and $x \in(0,1)$ (and the derivatives at 0 and 1 are defined as the unilateral derivatives).

(ii) We do not need Theorem 1 to prove that $\left(P_{1}\right)-\left(P_{4}\right)$ are $H$-ambivalent. It is clearly sufficient to apply Jarník's result from [J1] concerning (2).

In the following remark we present several consequences (which concern one-sided derivatives and Dini derivatives) of Kolář's [K1] result. Recall that $f \in C[0,1]$ is said to be a Besicovitch function if it has no one-sided derivative, finite or infinite, at all points. Further recall that $x \in(0,1)$ is said to be a knot point of $f \in C[0,1]$ if $\bar{D}^{+} f(x)=\bar{D}^{-} f(x)=\infty$ and $\underline{D}^{+} f(x)=\underline{D}^{-} f(x)=-\infty$.

Remark 3. (i) The observation of D. Preiss used in the proof of [K1, Corollary 11] (see also [Si, p. 460]), which shows that the set of Besicovitch functions is Haar null, also shows that the complement of the set

$$
A:=\left\{f \in C[0,1]: f_{+}^{\prime}(x)=\infty\right.
$$

for all $x$ from a set having the cardinality of the continuum\}

is Haar null.

(ii) Denote by $\left(P_{i}^{+}\right)$and $\left(P_{i}^{D}\right), 1 \leq i \leq 4$, the properties obtained from the properties $\left(P_{i}\right)$ by replacing the ordinary derivative $f^{\prime}$ by the right derivative $f_{+}^{\prime}$ and by the upper right Dini derivative $D^{+} f$, respectively. Then of course the properties $\left(P_{2}^{D}\right)$ and $\left(P_{4}^{D}\right)$ hold for all $f \in C[0,1]$. Further, by (i) above, $\left(P_{1}^{+}\right)$, $\left(P_{1}^{D}\right)$ and $\left(P_{2}^{+}\right)$hold for all $f \in C[0,1]$ except those belonging to a Haar null set. The remaining properties $\left(P_{3}^{+}\right),\left(P_{4}^{+}\right)$and $\left(P_{3}^{D}\right)$ are $H$-ambivalent: this can easily be proved using Lemma 4 as in the proof of Proposition 7 (i) below.

(iii) The set

$$
B:=\left\{f \in C[0,1]: f_{\text {ap }}^{\prime}(x) \in \mathbf{R} \text { exists at some } x \in(0,1)\right\}
$$

is Haar null by [K1]. The Denjoy-Khintchine theorem on approximate Dini derivatives ([Sa, Theorem 10.1]) immediately implies that the complement of the set

$$
\begin{aligned}
C:=\left\{f \in C[0,1]: D_{\text {ap }}^{+} f(x)=D_{\text {ap }}^{-} f(x)=\infty\right. \text { and } & \\
& \left.D_{\text {ap }+} f(x)=D_{\text {ap }-} f(x)=-\infty \quad \text { for almost all } x \in(0,1)\right\}
\end{aligned}
$$

is contained in $B$ and consequently is Haar null. Since it is easy to see that the set of all non-knot points of any $f \in C[0,1]$ is an $F_{\sigma}$ set, we easily deduce that the complement of the set

$$
\begin{aligned}
D:=\{f \in C[0,1]: \text { the set of non-knot } \\
\text { points of } f \text { is a first category and Lebesgue null set }\}
\end{aligned}
$$

is Haar null. Let us remark that the set $D$ is a residual subset of $C[0,1]$ (see e.g. G, Theorem 19.5.5 (b)]) and that an analogous result (G, Theorem 13.6.2]) concerning Brownian paths also holds (and can also be proved via the DenjoyKhintchine theorem). 


\section{Some Related RESUlts}

Proceeding as in the proof of Theorem 6, we obtain the following result.

Proposition 7. (i) For each $a \in(0,1)$, the set

$$
V:=\left\{f \in C[0,1]: f_{+}^{\prime}(a)=\infty \text { and } f_{-}^{\prime}(a)=-\infty\right\}
$$

is not Haar null.

(ii) The set

$$
W:=\left\{f \in C[0,1]: \text { ap- } \lim _{y \rightarrow x} \frac{|f(y)-f(x)|}{|y-x|}=\infty \text { for each } x \in(0,1)\right\}
$$

is not Haar null.

Proof. (Sketch.) (i) Let $K \subset C[0,1]$ be a compact set. Let $\omega$ be defined by (8) and choose a continuous increasing $\varphi \in \Phi$ for which (9) holds. Put $g_{1}(x):=$ $|\varphi(x-a)|, x \in[0,1]$. It is easy to show that $K+g_{1} \subset V$.

(ii) By [MZ, Theorem 1] there exists a function $g_{2} \in C[0,1]$ such that $\operatorname{ap} \lim _{y \rightarrow x} \frac{\left|g_{2}(y)-g_{2}(x)\right|}{\varphi(|y-x|)}=\infty$ for each $x \in(0,1)$. It is easy to show that $K+g_{1} \subset$ $W$.

Remark 4. (i) If the conjecture from $\mathrm{HZ}$ (which says that for each $g \in C[0,1]$ almost all trajectories of the one-dimensional Brownian motion belong to $W+g$ ) holds, then $C[0,1] \backslash W$ is Haar null.

(ii) The above proposition implies that the set

$$
B:=\left\{f \in C[0,1]: f_{+}^{\prime}(x)=\infty \text { and } f_{-}^{\prime}(x)=-\infty \text { at some } x \in(0,1)\right\}
$$

is not Haar null. Question 1 from [Z2], which asks whether $C[0,1] \backslash B$ is Haar null, is probably still open.

We conclude this article by a remark concerning Besicovitch functions.

Remark 5. As recalled in Remark 3 (i), the set of all Besicovitch functions from $C[0,1]$ is Haar null by $\mathrm{K} 1$. Question 2 from [Z2 asks whether the set of all Besicovitch functions is even Gaussian null in $C[0,1]$ (note that Gaussian nullness implies Haar nullness but not vice versa; see $[\mathrm{BL}]$ ). This question has a very simple (negative) answer. Indeed, J. Kolář K2] observed that the set $\beta+T$, where $\beta$ is a fixed Besicovich function and $T$ is the set of all $f \in C[0,1]$ which are increasing and analytic on $[0,1]$, clearly contains only Besicovitch functions and is not Gaussian null. This follows from the fact that $T$ is not Gaussian null. To prove this wellknown fact (mentioned just above Question 2 in $\mathrm{Z2}$ ) observe that the set of all functions of the form $f(x)=\sum_{n=1}^{\infty} a_{n} x^{n-1} 2^{-n} \quad\left(a_{n} \in[0,1]\right)$ is a cube in $C[0,1]$ in the sense of [BL, p. 142] and then use [BL, Proposition 6.27].

\section{REFERENCES}

[BL] Y. Benyamini, J. Lindenstrauss, Geometric Nonlinear Functional Analysis, Vol. 1, Colloquium publications (American Mathematical Society); v. 48, Providence, Rhode Island, 2000. MR 1727673 (2001b:46001)

[D] R. Dougherty, Examples of non-shy sets, Fund. Math. 144 (1994), 73-88. MR.1271479 (96c:43001)

[G] K.M. Garg, Theory of Differentiation, John Wiley, New York, 1998. MR.1641563 (2000c:26002)

$[\mathrm{H}]$ B. R. Hunt, The prevalence of continuous nowhere differentiable functions, Proc. Amer. Math. Soc. 122 (1994), 711-717. MR1260170(95d:26009) 
[HZ] P. Holický, L. Zajíček, Nondifferentiable functions, Haar null sets and Wiener measure, Acta Univ. Carolinae - Math. Phys. 41 (2000), 7-11. MR.1802330 (2001j:46058)

[J1] V. Jarník, Über die Differenzierbarkeit stetiger Funktionen, Fund. Math. 21 (1933), 48-58.

[J2] V. Jarník, Sur la dérivabilité des fonctions continues, Publications de la Fac. des Sc. de L'Univ. Charles 129 (1934), 9 pp.

[J3] V. Jarník, Sur la dérivée approximative unilatérale, Mem. Soc. R. Sci. Boheme, Cl. Sci. 9 (1934), 10 pp.

[K1] J. Kolářr, Porous sets that are Haar null, and nowhere approximately differentiable functions, Proc. Amer. Math. Soc. 129 (2001), 1403-1408. MR1814166 (2003d:28021)

[K2] J. Kolář, Private communication.

[LP] J. Lindenstrauss, D. Preiss, On Fréchet differentiability of Lipschitz maps between Banach spaces, Annals Math. 157 (2003), 257-288. MR1954267(2003k:46058)

[MZ] J. Malý, L. Zajíček, Approximate differentiation: Jarník points, Fund. Math. 140 (1991), 87-97. MR.1139090 (92m:26006)

[Sa] S. Saks, Theory of the integral, Monogr. Mat. VII, New York, 1937.

[Si] K. Simon, Some dual statements concerning Wiener measure and Baire category, Proc. Amer. Math. Soc. 106 (1989), 455-463. MR0961409 (89k:26003)

[Z1] L. Zajíček, The differentiability structure of typical functions in $C(0,1)$, Real Anal. Exchange 13 (1987/88), pp. 119, 113-116, 93.

[Z2] L. Zajíček, Differentiability properties of typical continuous functions, Real. Anal. Exchange 25 (1999/2000), 149-158.

Charles University, Faculty of Mathematics and Physics, Sokolovská 83, 18675 Prague 8, Czech Republic

E-mail address: zajicek@karlin.mff.cuni.cz 